\title{
HARMONIC MEASURE AND ESTIMATES OF GREEN'S FUNCTION
}

\author{
RICHARD A. HUNT
}

The study of exceptional sets for nontangential limits of harmonic functions has led to the problem of the equivalence of harmonic measure and surface measure on the boundary of Lipschitz domains. In this note we will review the development of these results. We will see how estimates of Green's function can be used to relate the measures and look at methods of obtaining the desired estimates.

If $u$ is positive and harmonic in the unit ball $B \subset E_{n+1}$, the Poisson integral representation leads easily to finite nontangential limits at almost every point of $\partial B$. We are interested in modifications and generalizations of this result.

In 1950, A. P. Calderón [2] proved the following: If $u$ is harmonic in the unit ball $B \subset E_{n+1}$ and for each point $Q \in E, E \subset \partial B, u$ is bounded in some truncated cone $\Gamma(Q)$ with vertex at $Q$, then $u$ has a finite nontangential limit at almost every $Q \in E$.

In Calderón's result, we may assume the cones $\Gamma(Q)$ are tangent to, and truncated by the more distant surface of, a fixed small ball with center at the origin. In that case, $\hat{B}=\cup_{Q \in E} \Gamma(Q)$ is a starlike Lipschitz domain. Calderón's result could be stated with the hypothesis that $u$ is harmonic and bounded in $\hat{\boldsymbol{B}}$. In 1962, L. Carleson [4] obtained the same conclusion as in Calderón's result, but with the hypothesis that $u$ is harmonic and bounded from below in $\hat{B}$. In his proof, Carleson introduced the harmonic measure associated with the domain $\hat{B}$. An estimate of Green's function for $\hat{B}$ was used to obtain results in terms of surface measure.

In 1964, K.-O. Widman [11] showed if $u$ is harmonic and positive in a domain $D$ with $\partial D \in C^{1+\varepsilon}, \varepsilon>0$, then $u$ has finite nontangential limits at almost every point of $\partial D$. As in the proof of Carleson, harmonic measure was used and an estimate of Green's function showed the exceptional set was of zero surface measure.

Following the general outline of Carleson's proof, Hunt and Wheeden [8] in 1970 proved that functions which are positive and harmonic in domains $D$ with $\partial D \in \operatorname{Lip}(1)$ have finite nontangential limits except on a set of harmonic measure zero. The fact that this exceptional set is also of surface measure zero is a consequence of the 1976 result of B. Dahlberg [6] that harmonic measure and surface measure are equivalent on the boundary of Lipschitz domains.

Let us briefly review a method to obtain the nontangential limits. We assume that $D \subset E_{n+1}, n \geqslant 2, \partial D \in \operatorname{Lip}(1)$ and $D$ is starlike about a point $\boldsymbol{P}_{\mathbf{0}}$. In particular, we assume there is a fixed truncated open cone $\Gamma$ which is contained in $D$ whenever it is positioned with vertex at $Q \in \partial D$ and axis

An address presented at the 745th meeting of the American Mathematical Society, Evanston, Illinois, April 16, 1977; received by the editors July 20,1977.

AMS (MOS) subject classifications (1970). Primary 31B25. 
along $Q P_{0}$. Unless stated otherwise, we assume $P_{0}$ is the origin.

If $u$ is positive and harmonic in $D$, we have

$$
u(t P)=\int_{\partial D} K(P, Q) u(t Q) d \omega^{P_{0}}(Q), \quad 0 \leqslant t<1,
$$

where $\omega^{P}$ denotes harmonic measure and $K(P, Q)$ is the Radon-Nikodym derivative, $d \omega^{P} / d \omega^{P_{0}}$. (The integral in (1) represents the solution of the Dirichlet problem in $D$ with continuous boundary values $u(t Q)$ for $Q \in \partial D$.) Since $K\left(P_{0}, Q\right) \equiv 1$, (1) shows that the measures $u(t Q) d \omega^{P_{0}}(Q)$ are uniformly bounded by $u\left(P_{0}\right) . K(P, Q)$ can be shown to be a continuous function of $Q \in \partial D$ for fixed $P \in D$. (See Hunt and Wheeden [9].) It follows that we may take weak limits in (1) to obtain the representation

$$
u(P)=\int_{\partial D} K(P, Q) d \mu(Q),
$$

where $\mu$ is a Borel measure on $\partial D$. If $d \mu=f d \omega^{P_{0}}+d s$, where $s$ is singular with respect to harmonic measure, then $u(P)$ approaches $f\left(Q_{0}\right)$ as $P$ approaches $Q_{0}$ nontangentially for a.e. $\left(d \omega^{P_{0}}\right) Q_{0} \in D$. This follows from a result on the differentiation of integrals and estimates of $K(P, Q)$. We will outline the proof.

$$
\Delta\left(Q_{0}, \eta\right)=\left\{Q \in \partial D:\left|Q-Q_{0}\right|<\eta\right\}
$$

will denote a "disk" in $\partial D$ with center $Q_{0} \in \partial D$ and radius $\eta>0$. For convenience, we set

$$
d \mu_{Q_{0}}(Q)=\left|f(Q)-f\left(Q_{0}\right)\right| d \omega^{P_{0}}(Q)+d s(Q) .
$$

A generalization of Lebesgue's theorem yields (see Besicovitch [1])

$$
\int_{\Delta\left(Q_{0}, \eta\right)} d \mu_{Q_{0}}(Q)=o\left(\omega^{P_{0}}\left(\Delta\left(Q_{0}, \eta\right)\right)\right), \quad \eta \rightarrow 0,
$$

for a.e. $\left(d \omega^{P_{0}}\right) Q_{0} \in \partial D$.

Let $\Gamma \subset D$ be a nontangential open cone with vertex at $Q_{0}$. For $P \in \Gamma$ with $\left|\boldsymbol{P}-Q_{0}\right|=r$, set $R_{0}=\Delta\left(Q_{0}, r\right)$ and $R_{j}=\Delta\left(Q_{0}, 2^{j} r\right)-\Delta\left(Q_{0}, 2^{j-1} r\right), j \geqslant 1$. Harnack's inequality and the maximum principle are used to obtain (see Hunt and Wheeden [8], [9])

(4) $\sup _{Q \in R_{f}} K(P, Q) \leqslant c_{j} / \omega^{P_{0}}\left(\Delta_{j}\right), \quad j \geqslant 0$, where $\sum_{j>0} c_{j} \leqslant C(\Gamma)<\infty$.

For fixed $\delta>0$ and any $P \in \Gamma$ with $\left|P-Q_{0}\right|=r \leq \delta$, choose $N$ such that $2^{N-1} r<\delta<2^{N} r$. Then

$$
\begin{aligned}
& \left|u(P)-f\left(Q_{0}\right)\right| \\
= & \left|\int_{\partial D} K(P, Q)\left(f(Q) d \omega^{P_{0}}(Q)+d s(Q)\right)-\int_{\partial D} K(P, Q) f\left(Q_{0}\right) d \omega^{P_{0}}(Q)\right| \\
< & \int_{\partial D} K(P, Q) d \mu_{Q_{0}}(Q) \\
< & \sum_{j=0}^{N} \int_{R_{j}} K(P, Q) d \mu_{Q_{0}}(Q)+\int_{\partial D-\Delta\left(Q_{0,} \delta\right)} K(P, Q) d \mu_{Q_{0}}(Q) .
\end{aligned}
$$


It follows from (3) and (4) that the sum of the integrals over the $R_{j}$ 's approaches zero (uniformly in $P \in \Gamma$ with $\left|P-Q_{0}\right|=r \leqslant \delta$ ) as $\delta$ approaches 0 . (4) also implies that for fixed $\delta>0$, the integral outside $\Delta\left(Q_{0}, \delta\right)$ approaches zero as $P$ approaches $Q_{0}, P \in \Gamma$.

Let us now see how the size of Green's function near $\partial D$ affects the ratio of harmonic measure and surface measure. The arguments are those of Dahlberg [6].

Green's function for $D \subset E_{n+1}, n \geqslant 2$, is

$$
G\left(P, P^{\prime}\right)=\frac{1}{\left|P-P^{\prime}\right|^{n-1}}-\int_{\partial D} K(P, Q) \frac{1}{\left|Q-P^{\prime}\right|^{n-1}} d \omega^{P_{0}}(Q) .
$$

Note that for fixed $P^{\prime}$,

$$
\int_{\partial D} K(P, Q) \frac{1}{\left|Q-P^{\prime}\right|^{n-1}} d \omega^{P_{0}}(Q)
$$

is a positive and harmonic function of $P \in D$ with boundary values $1 / \mid Q-$ $\left.P^{\prime}\right|^{n-1}, Q \in \partial D$.

For a fixed point $Q \in \partial D$, let $\Delta=\Delta(Q, r)$ and let $A=A(Q, r)$ be that point on the segment $Q P_{0}$ with $|A-Q|=C_{1} r$, where $C_{1}$ is a fixed constant. Let $B=\{P:|P-A| \leqslant 2 r\}$. The Lipschitz character of $\partial D$ allows us to choose $C_{1}$ large enough to insure that $B \subset D . \sigma(E)$ will denote the surface measure of $E \subset \partial D$. $C$ will denote various positive constants. We have

$$
C \frac{\omega^{P_{0}}(\Delta)}{\sigma(\Delta)} \leqslant \frac{G\left(A, P_{0}\right)}{r} \leqslant C \frac{\omega^{P_{0}}(\Delta)}{\sigma(\Delta)} .
$$

To see the first inequality in (5), we compare $G$ with Green's function for $B$ with pole at $A$. This yields

$$
G(P, A) \geqslant \frac{1}{|P-A|^{n-1}}-\frac{1}{(2 r)^{n-1}} \geqslant \frac{C}{r^{n-1}}, \quad|P-A| \leqslant r .
$$

Choose $t$ such that $t Q=A$. (Recall $P_{0}$ is the origin.) Then

$$
\{t P: P \in \Delta\} \subset\{P:|P-A|<\operatorname{tr}\} \subset\{P:|P-A| \leqslant r\} .
$$

The maximum principle then implies

$$
\omega^{P}(\Delta) \leqslant C r^{n-1} G(t P, A), \quad P \in D .
$$

Since $\sigma(\Delta) \approx r^{n}$ and $G\left(A, P_{0}\right)=G\left(P_{0}, A\right)$, we obtain the desired inequality by setting $P=P_{0}$.

To see the second inequality, note $G(P, A) \leqslant 1 /|P-A|^{n-1}$. Hence, $G(P, A) \leqslant(2 r)^{1-n}, \quad P \in \partial B$. Since $\omega^{P}(\Delta) \geqslant C>0, P \in \partial B$ (see, for example, [8, Lemma 2.1]), the maximum principle gives

$$
G(P, A) \leqslant C r^{1-n} \omega^{P}(\Delta), \quad P \in D-B .
$$

As before, we obtain the desired inequality by setting $P=P_{0}$.

We can now prove:

(6a) If $\inf _{r>0} G\left(A, P_{0}\right) / r>0$ for a.e. (do) $Q \in \partial D$, then $\omega^{P_{0}}(E)=0$ implies $\sigma(E)=0$.

From (5), we have that $\sigma(\Delta) \leqslant C_{Q} \omega^{P_{0}}(\Delta)$ for a.e. $(d \sigma) Q \in \partial D$. Since $\sigma(E)=\lim _{M \rightarrow \infty} \sigma\left(\left\{Q \in E: C_{Q} \leqslant M\right\}\right)$, we may assume $C_{Q} \leqslant M<\infty, Q \in E$. 
Given any $\varepsilon>0$, choose open $O \supset E$ such that $\omega^{P_{\mathrm{\theta}}}(O)<\varepsilon$. Each $Q \in E$ is the center of a disk $\Delta(Q) \subset O$. A Besicovitch type covering theorem (see [1]) then gives a countable subcollection $\left\{\Delta\left(Q_{j}\right)\right\}_{j \geqslant 1}$ such that

$$
E \subset \bigcup_{j>1} \Delta\left(Q_{j}\right) \subset O \text { and } \sum_{j>1} \chi_{\Delta\left(Q_{j}\right)} \leqslant C_{n+1} \chi_{\cup_{j>1} \Delta\left(Q_{j}\right)},
$$

where $\chi_{\Delta}$ is the characteristic function of $\Delta$ and $C_{n+1}$ is a constant which depends only on the Euclidean dimension. Then

$$
\begin{aligned}
\sigma(E) & \leqslant \sum_{j>1} \sigma\left(\Delta\left(Q_{j}\right)\right) \leqslant M \sum_{j>1} \omega^{P_{0}}\left(\Delta\left(Q_{j}\right)\right) \\
& \leqslant M C_{n+1} \omega^{P_{0}}\left(\bigcup_{j>1} \Delta\left(Q_{j}\right)\right) \leqslant M C_{n+1} \omega^{P_{0}}(O) \leqslant M C_{n+1} \varepsilon .
\end{aligned}
$$

Similarly, we have:

(6b) If $\sup _{r>0} G\left(A, P_{0}\right) / r<\infty$ for a.e. $\left(d \omega^{P_{0}}\right) Q \in \partial D$, then $\sigma(E)=0$ implies $\omega^{P_{0}}(E)=0$.

Let us now consider how to obtain the desired estimates of Green's function.

It is convenient to write $P=(x, y), x \in E_{n}, y$ real. We assume $D \subset E_{n+1}$ is bounded on the sides by $\{|x|=1\}$, on top by $\{y=1\}$, and on bottom by $\{(x, y): y=f(x)\}$, where $f \in \operatorname{Lip}(1)$. For $\left(x_{0}, f\left(x_{0}\right)\right)$ on the bottom part of $\partial D$, we redefine $A\left(\left(x_{0}, f\left(x_{0}\right)\right), r\right)$ to be the point with coordinates $\left(x_{0}, f\left(x_{0}\right)+\right.$ $C_{1} r$ ).

The first estimate we consider applies when $\partial D$ is bounded above near a point $\left(x_{0}, y_{0}\right)$ on the bottom part of $\partial D$ by a surface which is sufficiently close to its tangent plane at $\left(x_{0}, y_{0}\right)$. That is, let

$$
D_{\phi, \delta}=\left\{(x, y):|x|<\delta, \phi(x)<y<\phi_{0}(\delta)\right\},
$$

where $\phi(x)=\phi_{0}(|x|), \phi_{0}$ is continuous and nondecreasing on $[0,1)$ with $\phi_{0}(0)=0$. Moreover, we assume $\int_{|t|<1} \phi(t) /|t|^{n+1} d t<\infty$. If it is possible to reposition $D_{\phi, \delta}$ in such a way that the origin is moved to $\left(x_{0}, y_{0}\right)$ and the repositioned domain is contained in $D$, then

$$
\inf _{r>0} G\left(A, P_{0}\right) / r \geqslant C>0,
$$

where $A=A\left(\left(x_{0}, y_{0}\right), r\right)$.

To see this, we adopt the coordinate system of $D_{\phi, \delta}$ positioned with origin at $\left(x_{0}, y_{0}\right)$. The coordinates of $A$ become $(x, y)=\left(C_{1} r \sin \theta \cdot x^{\prime}, C_{1} r \cos \theta\right)$, where $\theta$ and $x^{\prime}$ are fixed with $0<\theta<\pi / 2$ and $\left|x^{\prime}\right|=1$. We will show $\inf _{0<y<\delta / 2} G\left((0, y), P_{0}\right) / y \geqslant C>0$. (The arguments could be carried out with $A$ in place of $(0, y)$ or we could use Harnack's inequality to obtain $G\left(A, P_{0}\right) \geqslant C G\left((0, r), P_{0}\right)$.)

We will need the auxillary function

$$
H(x, y)=\int_{|t|<\delta} \frac{\phi(t) y}{\left[(x-t)^{2}+y^{2}\right]^{(n+1) / 2}} d t .
$$

For $0<|x|<\delta / 2$, let 


$$
B=\{t:|t-(1+\phi(x) /|x|) x|<\phi(x)\} .
$$

Then $\phi(t) \geqslant \phi(x)$ for $t \in B$. We assume $\delta$ is small enough to insure $\phi_{0}(\delta / 2)$ $<\delta / 4$, so $B \subset\{|x|<\delta\}$. It follows that

$$
H(x, \phi(x)) \geqslant \phi(x) \int_{B} \frac{\phi(x)}{\left[(x-t)^{2}+(\phi(x))^{2}\right]^{(n+1) / 2}} d t \geqslant C_{0} \phi(x) .
$$

Hence, the harmonic function $C_{0} y-H(x, y)$ is nonpositive at $(x, \phi(x))$, $|x|<\delta / 2$. By replacing $\phi$ by a larger multiple of $\phi$, if necessary, we may assume that $T=\left\{\left(x, \phi_{0}(\delta / 2)\right):|x| \leqslant \delta / 2\right\}$ satisfies $T \subset D$. Then $G\left((x, y), P_{0}\right)$ is bounded from below on $T$ by a positive constant. Also, $C_{0} y-H(x, y)$ is bounded from above on $T$. The maximum principle then implies that a positive multiple of $C_{0} y-H(x, y)$ is majorized in $D_{\phi, \delta / 2}$ by $G\left((x, y), P_{0}\right)$. But

$$
\frac{C_{0} y-H(0, y)}{y} \geqslant C_{0}-\int_{|t|<8} \frac{\phi(t)}{t^{n+1}} d t \geqslant \frac{C_{0}}{2}
$$

if $\delta$ is sufficiently small. (Note that $C_{0}$ is independent of $\delta$.)

If $\partial D \in C^{1+\varepsilon}, \varepsilon>0$, the above estimate and (6a) yield that harmonic measure zero implies surface measure zero. For the sawtooth Lipschitz domains considered by Calderón [2] and Carleson [4], the same type of estimate works with $\phi(x)=\operatorname{dist}(x, E)$. This estimate of Green's function also appears in Calderón [3].

The technique of using a Poisson integral to estimate Green's function is not available for general Lipschitz domains. Dahlberg obtained the desired estimates in a very elegant way, which we shall outline.

First, note that the mean value theorem implies

$$
\sup _{r>0} \frac{G\left(A, P_{0}\right)}{C_{1} r} \leqslant \sup _{r>0} \frac{\partial G}{\partial y}\left(A, P_{0}\right) .
$$

To estimate $\partial G / \partial y$, we construct domains $D_{j} / D$ with $\partial D_{j}=\partial D$, except on the bottom part of $\partial D$ where we replace the $\operatorname{Lip}(1)$ boundary function $f$ of $D$ by a $C^{\infty}$ function. The functions $\partial G_{j} / \partial y$ are uniformly bounded from below on the common boundaries of $D_{j}$ and on the boundary of a small ball $B$ with center $P_{0}$. Also, $\partial G_{j} / \partial y \geqslant 0$ on the bottom part of $D_{j}$. The minimum principle for harmonic functions then implies $\partial G_{j} / \partial y \geqslant-C>-\infty$ in $D-B$. It follows that the harmonic function $\partial G / \partial y$ is also bounded from below in $D-B$, so the result of Hunt and Wheeden implies that $\partial G / \partial y$ has finite nontangential limits at a.e. $\left(d \omega^{P_{0}}\right) Q \in \partial D$. In particular, sup $r>0$. $\partial G$ $\left(A, P_{0}\right) / \partial y<\infty$ for a.e. $\left(d \omega^{P_{0}}\right) Q \in \partial D$, so (6b) and (7) may be used to obtain that surface measure zero implies harmonic measure zero.

We now have $\omega^{P_{0}}(E)=\int_{E} k d \sigma$. Essentially, $k$ is given by the boundary values of $\partial G / \partial y$. Recall that $G\left(P, P_{0}\right)=\left|P-P_{0}\right|^{1-n}+h(P)$, where $h$ is harmonic in $D$. Hence, $\partial G\left(P, P_{0}\right) / \partial y=b(P)+\partial h(P) / \partial y$, where $b(P)$ is uniformly bounded for $P$ near $\partial D$. Then 


$$
\begin{aligned}
\int_{\partial D} k^{2} d \sigma & =\int_{\partial D} k d \omega^{P_{0}} \approx \int_{\partial D}\left(b+\frac{\partial h}{\partial y}\right) d \omega^{P_{0}} \\
& \approx \int_{\partial D} b d \omega^{P_{0}}+\frac{\partial h}{\partial y}\left(P_{0}\right)<\infty .
\end{aligned}
$$

It follows that

$$
\omega^{P_{0}}(E)=\int_{E} k d \sigma \leqslant\left(\int_{\partial D} k^{2} d \sigma\right)^{1 / 2}(\sigma(E))^{1 / 2} .
$$

The uniform estimate given by (9) can be used to show that $\omega^{P_{o}}(E)=0$ implies $\sigma(E)=0$. However, Dahlberg also obtained a local version of (8) which gives even sharper results. Dahlberg showed

$$
\frac{1}{\sigma(\Delta)} \int_{\Delta} k^{2} d \sigma \leqslant C\left(\frac{1}{\sigma(\Delta)} \int_{\Delta} k d \sigma\right)^{2}
$$

This inequality implies that $\int_{\partial D} k^{p} d \sigma<\infty$ for some $p>2$. (See Gehring [7].) Also, it shows the measures $d \omega^{P_{0}}$ and $d \sigma$ are comparable in the sense of Coifman and Fefferman [5]. It follows that

$$
\begin{cases}\omega^{P_{0}}(E) \leqslant C(\sigma(E))^{\alpha} & \text { for some } \alpha>\frac{1}{2}, \text { and } \\ \sigma(E) \leqslant C\left(\omega^{P_{0}}(E)\right)^{\beta} & \text { for some } \beta>0 .\end{cases}
$$

In a certain sense, (11) is a best possible result. (See [6].) That is, consider the harmonic measure in $D=\{z:|z|<1, \operatorname{Im} z>0\}$ of $\Delta=\{|z|<\delta\} \cap \partial D$. We have $\omega_{D}^{z_{0}}(\Delta) \approx C \delta, z_{0}=i / 2$. (See [10, p. 55].) The transformation $z \rightarrow z^{\gamma}$ for fixed $\gamma, 0<\gamma<2$, transforms $D$ into a Lipschitz domain $D_{\gamma} . \Delta$ is transformed into a "disk" $\Delta_{\gamma}$ of radius $\delta^{\gamma}$, with

$$
\omega_{D}^{z \gamma}\left(\Delta_{\gamma}\right) \approx \omega_{D}^{z_{0}}(\Delta) \approx C \delta \approx C\left(\sigma\left(\Delta_{\gamma}\right)\right)^{1 / \gamma} .
$$

This shows the sharpness of the exponents in (11) and how they depend on the Lipschitz constant of the domain.

For a fixed Lipschitz domain it is easy to see that

$$
\omega^{P_{0}}(\Delta) \leqslant C(\sigma(\Delta))^{\alpha} \text { and } \sigma(\Delta) \leqslant C\left(\omega^{P_{0}}(\Delta)\right)^{\beta} \text {, }
$$

for disks $\Delta$, where $\alpha$ and $\beta$ depend on the Lipschitz constants. It would be interesting to know if the same $\alpha$ and $\beta$ work for sets other than disks.

\section{REFERENCES}

1. A. S. Besicovitch, $A$ general form of the covering principle and relative differentiation of additive functions. II, Proc. Cambridge Philos. Soc. 42 (1946), 1-10.

2. A. P. Calderon, On the behavior of harmonic functions at the boundary, Trans. Amer. Math. Soc. 68 (1950), 47-54.

3. On a theorem of Marcinkiewicz and Zygmund, Trans. Amer. Math. Soc. 68 (1950), $55-61$.

4. L. Carleson, On the existence of boundary values for harmonic functions in several variables, Ark. Mat 4 (1962), 393-399.

5. R. R. Coifman and C. Fefferman, Weighted norm inequalities for maximal functions and singular integrals, Studia Math. 51 (1974), 241-250. 
6. B. E. J. Dahlberg, On estimates of harmonic measure, Arch. Rational Mech. Anal. 65 (1977), 275-288.

7. F. W. Gehring, The $L^{P}$-integrability of the partial deriviatives of a quasiconformal mapping, Acta Math. 130 (1973), 265-277.

8. R. A. Hunt and R. L. Wheeden, On the boundary values of harmonic functions, Trans. Amer. Math. Soc. 132 (1968), 307-322.

9. Positive harmonic functions on Lipschitz domains, Trans. Amer. Math. Soc. 147 (1970), 507-527.

10. M. Ohtsuka, Dirichlet problem, extremal length, and prime ends, Van Nostrand, Reinhold, New York, 1970.

11. K.-O. Widman, On the boundary values of harmonic functions in $\mathbf{R}^{3}$, Ark. Mat. 5 (1964), 221-230.

Department of Mathematics, Purdue University, West Lafayette, Indiana 47907 